\title{
High-angular resolution observations of methanol in the infrared dark cloud core G11.11-0.12P1 ${ }^{\star}, \star \star$
}

\author{
L. Gómez ${ }^{1, \star \star \star}$, F. Wyrowski ${ }^{1}$, T. Pillai ${ }^{2}$, S. Leurini $^{1}$, and K. M. Menten ${ }^{1}$ \\ ${ }^{1}$ Max-Planck-Institut für Radioastronomie, Auf dem Hügel 69, 53121 Bonn, Germany \\ e-mail: [lgomez, wyrowski, sleurini, kmenten]@mpifr.de \\ 2 California Institute of Technology, MC 249-17, 1200 East California Boulevard, Pasadena, CA 91125, USA \\ e-mail: tpillai@astro.caltech.edu
}

Received 14 December 2010 / Accepted 7 March 2011

\begin{abstract}
Recent studies suggest that infrared dark clouds (IRDCs) have the potential of harboring the earliest stages of massive star formation and indeed evidence for this is found toward distinct regions within them. We present a study with the Plateau de Bure Interferometer of a core in the archetypal filamentary IRDC G11.11-0.12 of a few arcsecond resolution to determine its physical and chemical structures. The data consist of continuum and line observations covering the $\mathrm{C}^{34} \mathrm{~S} 2 \rightarrow 1$ line and the methanol $2_{k} \rightarrow 1_{k} v_{t}=0$ lines at $3 \mathrm{~mm}$ and the methanol $5_{k} \rightarrow 4_{k} v_{t}=0$ lines at $1 \mathrm{~mm}$. Our observations show extended emission in the continuum at 1 and $3 \mathrm{~mm}$. The methanol $2_{k} \rightarrow 1_{k} v_{t}=0$ emission has three maxima extending over a $1 \mathrm{pc}$ scale (when merged with single-dish short-spacing observations); one of the maxima is spatially coincident with the continuum emission. The fitting results show an enhanced methanol fractional abundance $\left(\sim 3 \times 10^{-8}\right)$ at the central peak with respect to the other two peaks, where it decreases by about an order of magnitude $\left(\sim 4-6 \times 10^{-9}\right)$. Evidence of extended $4.5 \mu \mathrm{m}$ emission, "wings" in the $\mathrm{CH}_{3} \mathrm{OH} 2_{k} \rightarrow 1_{k}$ spectra, and $\mathrm{CH}_{3} \mathrm{OH}$ abundance enhancements point to the presence of an outflow in the east-west direction. In addition, we find a gradient of $\sim 4 \mathrm{~km} \mathrm{~s}^{-1}$ in the same direction, which we interpret as being produced by an outflow(s)-cloud interaction.
\end{abstract}

Key words. ISM: abundances - ISM: individual objects: G11.11-0.12P1 - ISM: molecules - techniques: interferometric stars: formation

\section{Introduction}

The objects now termed infrared dark clouds (IRDCs) were first identified by Perault et al. (1996) and Egan et al. (1998) in images of the Galactic plane made with the Infrared Space Observatory (ISO) and the Midcourse Space Experiment (MSX) satellite, respectively. IRDCs have significant extinctions even at 8 and $15 \mu \mathrm{m}$ and are, thus, seen in silhouette against the bright, diffuse, mid-infrared Galactic emission. Millimeter and (sub)millimeter molecular studies reveal that some clumps within IRDCs have column densities of $>10^{23} \mathrm{~cm}^{-2}$, volume gas densities of $n>10^{5} \mathrm{~cm}^{-3}$, and temperatures $T<20 \mathrm{~K}$ (e.g., Carey et al. 1998; Pillai et al. 2006a). Several studies suggest that IRDCs have the potential of harboring the earliest stages of massive star formation and indeed evidence for this is found toward distinct regions within them (Rathborne et al. 2005; Pillai et al. 2006b; Wang et al. 2006).

Pillai et al. (2006a) mapped the $(J, K)=(1,1)$ and $(2,2)$ inversion transitions of ammonia $\left(\mathrm{NH}_{3}\right)$ in the IRDC G11.11-0.12

\footnotetext{
* Based on observations carried out with the IRAM Plateau de Bure Interferometer and the IRAM $30 \mathrm{~m}$ telescope. IRAM is supported by INSU/CNRS (France), MPG (Germany), and IGN (Spain).

$\star \star$ Fits images associated with the $1 \mathrm{~mm}$ and $3 \mathrm{~mm}$ continuum maps of Fig. 1 and the integrated intensity maps of Figs. 3-5, and 9 can be queried from the CDS via anonymous ftp to

cdsarc.u-strasbg.fr $(130.79 .128 .5)$ or via

http://cdsweb.u-strasbg.fr/cgi-bin/qcat?]/A+A/529/A161

$\star \star \star$ Member of the International Max Planck Research School (IMPRS) for Astronomy and Astrophysics at the Universities of Bonn and Cologne.
}

with the Effelsberg $100 \mathrm{~m}$ telescope and found gas temperatures of the order of $15 \mathrm{~K}$ for several clumps within this cloud. The left panel of Fig. 1 shows an overview of the region. Furthermore, Pillai et al. (2006b) reported the detection of $6.7 \mathrm{GHz}$ class II methanol $\left(\mathrm{CH}_{3} \mathrm{OH}\right)$ and $22.2 \mathrm{GHz}$ water $\left(\mathrm{H}_{2} \mathrm{O}\right)$ masers in the IRDC core G11.11-0.12P1 (hereafter G11.11P1). Both $\mathrm{CH}_{3} \mathrm{OH}$ and $\mathrm{H}_{2} \mathrm{O}$ masers are known as tracers of massive star formation. They found that these masers were associated with a SCUBA dust continuum peak (P1; Carey et al. 2000) and ascribed the kinematics of the methanol masing spots to a maser amplification from a Keplerian disk.

Henning et al. (2010) observed this filamentary IRDC with the PACS (at 70, 100, and $160 \mu \mathrm{m}$ ) and the SPIRE (250, 350, and $500 \mu \mathrm{m})$ instruments onboard the Herschel Space Observatory, with resolutions in the range of $\sim 6^{\prime \prime}-40^{\prime \prime}$. For G11.11P1, they obtain from a modified blackbody fit to the PACS data, a dust temperature of $24 \mathrm{~K}$, a luminosity of $1346 L_{\odot}$, and a mass of $240 M_{\odot}$. Pillai et al. (2006a) and Leurini et al. (2007b) used a two-component model to fit single-dish observations of $\mathrm{NH}_{3}$ and $\mathrm{CH}_{3} \mathrm{OH}$ spectra, respectively, and derived temperatures of $\sim 15-18 \mathrm{~K}$ for the extended component (with size of $\sim 20^{\prime \prime}$ corresponding to $0.35 \mathrm{pc}$ ) and $\sim 47-60 \mathrm{~K}$ for the inner component (with a size of $\sim 3^{\prime \prime}$ corresponding to $0.05 \mathrm{pc}$ ).

Methanol, which is a slightly asymmetric top molecule, has been used to derive physical parameters such as the density and temperature of IRDCs (Leurini et al. 2007b), highmass protostellar objects (e.g., Leurini et al. 2007a), and massive young stars (e.g., van der Tak et al. 2000), as well as low-mass protostellar systems (Kristensen et al. 2010). In particular, 

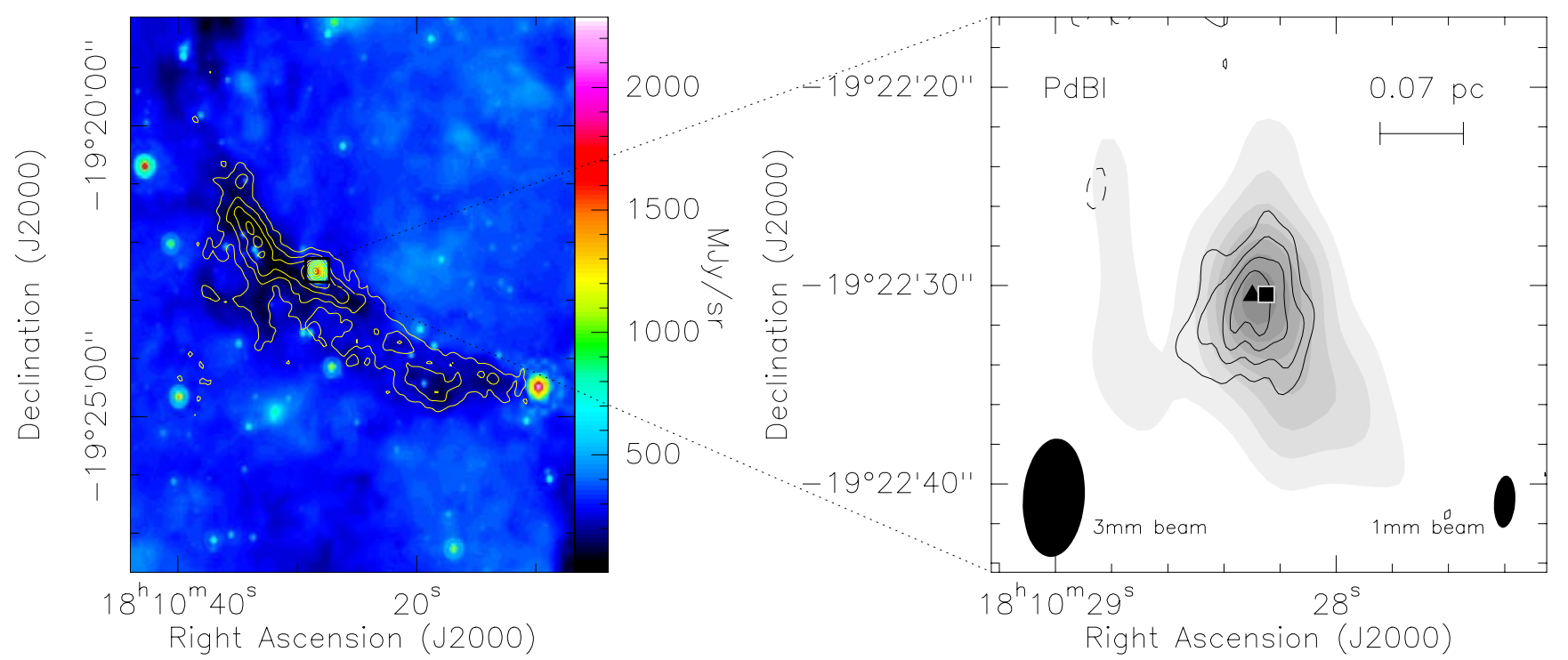

Fig. 1. Left: SCUBA $850 \mu \mathrm{m}$ (Carey et al. 2000) contours overlaid on the MIPSGAL $24 \mu \mathrm{m}$ image of the IRDC G11.11-0.12. Right: PdBI 1.2 mm continuum emission (contours) of G11.11P1 overlaid on the $3.1 \mathrm{~mm}$ continuum emission (grey scale image). First contour and contour spacing for the $3.1 \mathrm{~mm}$ emission are $0.9 \mathrm{mJy}^{-}$beam $^{-1}(3 \sigma)$, the dotted contours show the negative emission $(-3 \sigma)$; the synthesized beam $\left(6{ }^{\prime \prime} 0 \times 33^{\prime \prime} 1\right.$; $\left.\mathrm{PA}=176^{\circ}\right)$ is shown in the bottom left corner. First contour and contour spacing for the $1.2 \mathrm{~mm}$ emission are $6.3 \mathrm{mJy}^{\circ}$ beam ${ }^{-1}(3 \sigma)$, the dashed contours show the negative emission $(-3 \sigma)$; the synthesized beam $\left(2^{\prime \prime} 6 \times 11^{\prime \prime} 1\right.$; PA $\left.=176^{\circ}\right)$ is shown in the bottom right corner. The filled triangle at $\mathrm{RA}=18^{\mathrm{h}} 10^{\mathrm{m}} 28^{\mathrm{s}} .29$, Dec $=-19^{\circ} 22^{\prime} 30^{\prime} 5$ and the filled square at RA $=18^{\mathrm{h}} 10^{\mathrm{m}} 28^{\mathrm{s}} .25$, Dec $=-19^{\circ} 22^{\prime} 30^{\prime} \cdot 45$ (J2000) indicate the water and (integrated emission) methanol masers, respectively, reported by Pillai et al. (2006b).

van der Tak et al. (2000) carried out a study toward 13 massive star-forming regions and found three types of $\mathrm{CH}_{3} \mathrm{OH}$ abundance $\left(X_{\mathrm{CH}_{3} \mathrm{OH}} \equiv N_{\mathrm{CH}_{3} \mathrm{OH}} / N_{\mathrm{H}_{2}}\right)$ profiles: $X_{\mathrm{CH}_{3} \mathrm{OH}} \sim 10^{-9}$ for the coldest sources, from $10^{-9}$ to $10^{-7}$ for warmer sources, and $10^{-7}$ for hot cores. $\mathrm{CH}_{3} \mathrm{OH}$ has also been associated with outflows where the methanol abundance enhancements have been found to be a factor as large as 400-1000 (e.g., Bachiller et al. 1995; Garay et al. 2002; Kristensen et al. 2010).

In this paper, we present arcsecond-resolution millimeter continuum and line observations toward G11.11P1 to determine its physical and chemical structure. In Sect. 2, we describe our observations carried out with the IRAM Plateau de Bure Interferometer. In Sect. 3, we present continuum and line results together with the analysis. The discussion is presented in Sect. 4 and the summary is given in Sect. 5.

\section{Observations and data reduction}

\subsection{IRAM PdBI observations}

G11.11P1 was observed with the IRAM six element array Plateau de Bure Interferometer (PdBI; Guilloteau et al. 1992) in D and C configurations in 2005 and 2006, respectively.

During the 2006 observations, one antenna was equipped with the prototype New Generation Receiver. Because of a difference in the frequency scheme of this receiver, visibilities obtained in the image sideband in this antenna were discarded. The receivers were tuned single side-band at $3 \mathrm{~mm}$ and double sideband at $1 \mathrm{~mm}$. The $3 \mathrm{~mm}$ receivers were centered at $96.64 \mathrm{GHz}$ and the $1 \mathrm{~mm}$ receivers at $241.81 \mathrm{GHz}$ (see Table 1 ). At $3 \mathrm{~mm}$, the $\mathrm{C}^{34} \mathrm{~S} 2 \rightarrow 1$ line and the $\mathrm{CH}_{3} \mathrm{OH} 2_{k} \rightarrow 1_{k} v_{t}=0$ lines were covered by using two correlator units of $80 \mathrm{MHz}$ with a spectral resolution of $0.3125 \mathrm{MHz}\left(0.97 \mathrm{~km} \mathrm{~s}^{-1}\right)$ and by two correlator units of $320 \mathrm{MHz}$. The latter, which are free of lines, were used to produce the continuum image with a total bandwith of $\sim 450 \mathrm{MHz}$. At $1 \mathrm{~mm}$, the $\mathrm{CH}_{3} \mathrm{OH} 5_{k} \rightarrow 4_{k} v_{t}=0$ lines were observed with two units of $160 \mathrm{MHz}$ with a spectral resolution of
Table 1. Parameters for the IRAM PdBI observations.

\begin{tabular}{lc}
\hline \hline Parameter & Value \\
\hline Pointing center & RA $(\mathrm{J} 2000)=18^{\mathrm{h}} 10^{\mathrm{m}} 28^{\mathrm{s}} .24$ \\
& Dec $(\mathrm{J} 2000)=-19^{\circ} 22^{\prime} 30^{\prime} .5$ \\
Number of antennas & 6 \\
Baseline range & $24-176 \mathrm{~m}$ \\
Band center & 96.64 and $241.81 \mathrm{GHz}$ \\
Primary HPBW & $52^{\prime \prime}$ at $96.64 \mathrm{GHz}$ \\
& $21^{\prime \prime}$ at $241.81 \mathrm{GHz}$ \\
Synthesised HPBW & $6{\text { 6.' } 0 \times 33^{\prime \prime} 1 \text { at } 96.64 \mathrm{GHz}}^{\prime \prime}(1.17 \mathrm{Jy}$ at $96.64 \mathrm{GHz}$ \\
Primary flux density calibrator: & $1.81 \mathrm{GHz}$ \\
MWC 349 & $2.03 \mathrm{Jy}$ at $241.81 \mathrm{GHz}$ \\
\hline
\end{tabular}

1.250 MHz (1.55 $\left.\mathrm{km} \mathrm{s}^{-1}\right)$. The remaining units of 80,160 , and $320 \mathrm{MHz}$ were placed in such a way that a frequency range free of lines could be used to measure the continuum flux. The total bandwidth of both sidebands was $\sim 700 \mathrm{MHz}$.

The phase and amplitude were calibrated with observations of the object 1730-130. The bandpass calibration was done by observing 3C 273. MWC 349 was used as a primary flux calibrator of the 3 and $1 \mathrm{~mm}$ data (see Table 1). We estimate the final flux-density accuracy to be $\sim 5 \%$ and $10 \%$ for the $3 \mathrm{~mm}$ and $1 \mathrm{~mm}$ data, respectively. Continuum images were subtracted from the line data in the visibility plane. The combination of the $\mathrm{C}$ and $\mathrm{D}$ configurations provides angular scales in the ranges 1 ." $8-12$ ". $6(1 \mathrm{~mm})$ and 4".4-32.'5 (3 mm), i.e., providing information on spatial scales of $0.03-0.22 \mathrm{pc}$ and $0.08-0.57 \mathrm{pc}$, respectively.

The calibration and data reduction were completed using the GILDAS package ${ }^{1}$ at IRAM Grenoble. Images were created with natural weighting and CLEANed using the standard Högbom algorithm.

${ }^{1}$ http://wWw.iram.fr/IRAMFR/GILDAS 
Table 2. Parameters of the observed continuum emission.

\begin{tabular}{|c|c|c|c|c|c|c|}
\hline \multirow{2}{*}{$\begin{array}{r}\text { Frequency } \\
(\mathrm{GHz})\end{array}$} & \multicolumn{2}{|c|}{ Peak Intensity Position } & \multirow{2}{*}{$\begin{array}{l}\text { Peak Intensity } \\
\left(\mathrm{mJy}^{-} \text {beam }\right.\end{array}$} & \multirow{2}{*}{$\begin{array}{c}\text { Flux density }^{a} \\
(\mathrm{mJy})\end{array}$} & \multirow[t]{2}{*}{ Deconvolved angular size ${ }^{b}$} & \multirow{2}{*}{$\begin{array}{r}\operatorname{Mass}^{c} \\
\left(M_{\odot}\right)\end{array}$} \\
\hline & RA (J2000) & Dec (J2000) & & & & \\
\hline 96.6 & $18^{\mathrm{h}} 10^{\mathrm{m}} 28^{\mathrm{s}} .28$ & $-19^{\circ} 22^{\prime} 30^{\prime} 5$ & 7.0 & 20.2 & $\left(8{ }^{\prime \prime} 9 \pm 0.6\right) \times\left(4^{\prime \prime} \cdot 4 \pm 00^{\prime} 5\right) ;+24^{\circ} \pm 7^{\circ}$ & 37 \\
\hline 240.3 & $18^{\mathrm{h}} 10^{\mathrm{m}} 28^{\mathrm{s}} .29$ & $-19^{\circ} 22^{\prime} 30^{\prime} 5$ & 30.8 & 208.6 & $\left(4 \prime^{\prime \prime} 9 \pm 0 . \prime 6\right) \times\left(3{ }^{\prime \prime} 9 \pm 00^{\prime} 5\right) ;+165^{\circ} \pm 36^{\circ}$ & 13 \\
\hline
\end{tabular}

Notes. ${ }^{(a)}$ Flux density was obtained by summing the continuum emission above $3 \sigma$ level within a polygon encompassing the core.

${ }^{(b)}$ Major axis $\times$ minor axis, at $F W H M$; position angle of major axis from fits of elliptical Gaussian.

(c) Assuming a distance of $3.6 \mathrm{kpc}$ and dust temperature of $60 \mathrm{~K}$.

\subsection{IRAM $30 \mathrm{~m}$ short-spacing observations}

G11.11P1 was observed in the $2_{k} \rightarrow 1_{k} v_{t}=0(3 \mathrm{~mm})$ and $5_{k} \rightarrow 4_{k}(1 \mathrm{~mm}) \mathrm{CH}_{3} \mathrm{OH}$ bands with the IRAM $30 \mathrm{~m}$ telescope. An area of $90^{\prime \prime} \times 90^{\prime \prime}$ was mapped with the SIS B100 receiver at $3 \mathrm{~mm}$ and $66^{\prime \prime} \times 66^{\prime \prime}$ with the HERA receiver (Schuster et al. 2004) at $1 \mathrm{~mm}$ with a bandwidth of $140 \mathrm{MHz}$. Sampling at $3 \mathrm{~mm}$ was of $15^{\prime \prime}$, while at $1 \mathrm{~mm}$ it was of $6^{\prime \prime}$. A conversion from antenna temperature to main-beam brightness temperature $\left(T_{\mathrm{MB}}\right)$ was performed by using a beam efficiency of 0.78 at $3 \mathrm{~mm}$ and 0.52 at $1 \mathrm{~mm}$. The rms at $1 \mathrm{~mm}$ is $0.3 \mathrm{~K}$ and at $3 \mathrm{~mm}$ is $0.02 \mathrm{~K}$. The data were reduced with the CLASS program, which is part of the GILDAS software package.

The $3 \mathrm{~mm}$ single-dish data are used to create short-spacing pseudo-visibilities; these are then merged with the interferometric observations. All data are imaged and deconvolved together. The processing was performed in GILDAS/MAPPING following standard procedures of the UV_SHORT task (see Rodríguez-Fernández et al. $2008^{2}$ for details of the pseudovisitbility technique). In Sect. 3.3, we present the result of recovering the short-spacing information for the $3 \mathrm{~mm}$ data.

Unfortunately, we were unable to merge the interferometric and single-dish observations for the $1 \mathrm{~mm}$ data because of the poor signal-to-noise ratio of the latter set of data. However, we used the $1 \mathrm{~mm}$ single-dish data in the following sections to help in the analysis and interpretation of this core.

\section{Results and analysis}

\subsection{PdBI: Continuum emission}

\subsubsection{Morphology and core size}

The right panel of Fig. 1 presents the continuum images at 1 and $3 \mathrm{~mm}$ of G11.11P1. At the resolution of both images, the emission is extended; the peak intensity positions coincide with each other and with the maser positions as well. The morphology at $3 \mathrm{~mm}$ resembles that previously imaged with the Berkeley-Illinois-Maryland-Association (BIMA) interferometer by Pillai et al. (2006b) with a slightly lower resolution $\left(8, .3 \times 33^{\prime \prime} 9\right)$.

In Table 2, we list the parameters of the continuum emission. Deconvolved sizes were obtained by fitting two-dimensional Gaussians to the continuum maps and yielding a core size of $\sim 0.16 \mathrm{pc} \times 0.08 \mathrm{pc}$ with a PA $24^{\circ}$ at $3 \mathrm{~mm}$ and $\sim 0.09 \mathrm{pc} \times$ $0.07 \mathrm{pc}$ with a PA $165^{\circ}$ at $1 \mathrm{~mm}$. We assumed a kinematic distance of $3.6 \mathrm{kpc}$.

\footnotetext{
2 http://iram.fr/GENERAL/reports/

IRAM_memo_2008-2-short-spacings.pdf
}

\subsubsection{Mass}

Assuming that the mm continuum is mainly due to optically thin dust emission, we estimate the gas mass, $M_{\mathrm{g}}$, using the expression (Hildebrand 1983)

$M_{\mathrm{g}}=\frac{F_{v} D^{2} R_{\mathrm{gd}}}{\kappa_{v} B_{v}\left(T_{\mathrm{d}}\right)} M_{\odot}$

where $F_{v}$ is the observed integrated flux density in Jy, $D$ is the distance, $R_{\mathrm{gd}}$ is the gas-to-dust mass ratio, $\kappa_{v}$ is the dust opacity coefficient, and $B_{v}\left(T_{\mathrm{d}}\right)$ is the Planck function at the dust temperature $\left(T_{\mathrm{d}}\right)$. The $1 \mathrm{~mm}$ flux density was obtained by summing the $1 \mathrm{~mm}$ continuum emission above $3 \sigma\left(>6 \mathrm{mJy}\right.$ beam $\left.^{-1}\right)$ level within a polygon encompassing the core, and the integrated flux density is $\sim 209 \mathrm{mJy}$. We assume a gas-to-dust mass ratio of 100, and adopt a $\kappa_{v}=1 \mathrm{~cm}^{2} \mathrm{~g}^{-1}$ (Ossenkopf \& Henning 1994), at $1 \mathrm{~mm}$, for an MRN (Mathis et al. 1977) graphite-silicate grain mixture with thick ice mantles, at a gas density of $10^{6} \mathrm{~cm}^{-3}$. If we use $T_{\mathrm{d}}=60 \mathrm{~K}$ (Leurini et al. 2007b; Pillai et al. 2006a), a proper temperature for a region where methanol emission is produced, a mass of $13 M_{\odot}$ at $1 \mathrm{~mm}$ is obtained for G11.11P1 (see Table 2). This is equivalent to adopting a dust opacity coefficient of $k_{v}=0.1\left(v / 10^{12} \mathrm{~Hz}\right)^{\beta}$ (Beckwith et al. 1990), which includes the gas contribution to the total mass for a $R_{\mathrm{gd}}=100$, with $\beta=1.6$. Given the uncertainties in the models of dust opacities and variation of the gas-to-dust ratio, $\beta$ is within the errors comparable to the upper limit we obtain in Sect. 3.1.3.

Comparing the flux from the Bolocam Galactic Plane Survey (BGPS ${ }^{3}$; Aguirre et al. 2011) $1.1 \mathrm{~mm}$ with the PdBI $1.2 \mathrm{~mm}$ continuum flux, we find that the interferometric observations filter out about $75 \%$ of the emission.

To make a rough mass estimate of the core from the $3 \mathrm{~mm}$ data, all parameters remain the same as with the $1 \mathrm{~mm}$ data except for the flux density, $\sim 20 \mathrm{mJy}$, and $\kappa_{v}=0.2 \mathrm{~cm}^{2} \mathrm{~g}^{-1}$ (extrapolating from Table 1 Col. 9 in Ossenkopf \& Henning 1994). We obtain a core mass of $37 M_{\odot}$.

\subsubsection{Density, temperature, and spectral indeces}

For deeper insight into the source structure, we analyzed the $1 \mathrm{~mm}$ and $3 \mathrm{~mm}$ continuum data in the $u-v$ domain avoiding the cleaning and $u-v$ sampling effects. Figure 2 shows the averaged amplitudes averaged over a concentric annulus (from the continuum peak positions) versus deprojected $u-v$ distance in units of wavelength. The amplitudes were averaged vectorially and the $3 \mathrm{~mm} u-v$ amplitudes in Jy were scaled by a factor of 15 to match the $1 \mathrm{~mm}$ visibilities. Error bars are $1 \sigma$ statistical errors based on the standard deviation in the mean of the data points in the bin.

Since the index of the power law in $u-v$ distance is related to the index of the power law in radial distance, $r$, following

3 http://irsa.ipac.caltech.edu/data/BOLOCAM_GPS/ 


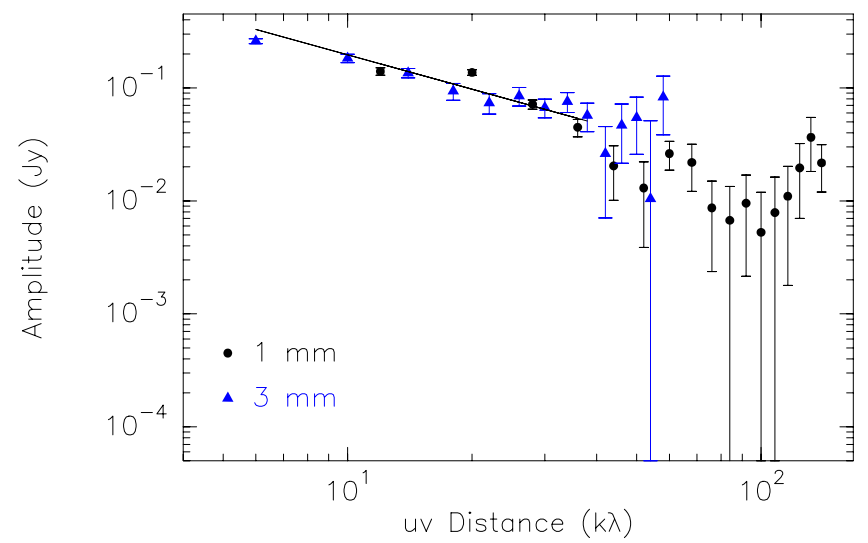

Fig. 2. Continuum emission from G11.11P1 at $3.1 \mathrm{~mm}$ (filled triangles) and $1.2 \mathrm{~mm}$ (filled circles) in the Fourier domain. Amplitudes are averaged in bins of deprojected $u-v$ distance from the continuum intensity peaks. The $3.1 \mathrm{~mm} u-v$ amplitudes have been scaled by a factor of 15 . The straight solid line represents the least squares fit to the data. Error bars represent $1 \sigma$ statistical errors based on the standard deviation in the mean of the data points in the bin.

Looney et al. (2003), we can write $F(r) \propto r^{-(p+q)+1} \rightarrow V(s) \propto$ $s^{(p+q)-3}$, where $F(r)$ is the flux density in the image domain, $V(s)$ is the Fourier transform of the flux density, $s$ is $u-v$ distance and, $p$ and $q$ are the power-law indices of the density $\left(n \propto r^{-p}\right)$ and temperature structure $\left(T \propto r^{-q}\right)$, respectively.

The least squares fit (see Fig. 2) to both the $1 \mathrm{~mm}$ and $3 \mathrm{~mm}$ data yields a slope of $-1.01 \pm 0.02$, corresponding to $p+q \sim 2.0$. Points starting at $40 k \lambda$, which corresponds to an angular scale of $\sim 6^{\prime \prime}(0.1 \mathrm{pc})$, were excluded from the fit because the signalto-noise ratio gets very low. If the index $q=0.4$ (Goldreich \& Kwan 1974), the density profile index is 1.6.

We estimate a mean volume gas density of the core $(n=$ $\frac{M_{\mathrm{g}}}{\frac{4}{3} \pi R^{3} \mu_{\mathrm{H}_{2}} m_{\mathrm{H}}}$, where $R$ is the core radius) of $7 \times 10^{5} \mathrm{~cm}^{-3}$ using the mass derived from the dust observations at $1 \mathrm{~mm}\left(13 M_{\odot}\right)$, and the geometric mean of the deconvolved linear size as the core $\operatorname{radius}\left(R=\sqrt{\left(F W H M_{\text {maj }} / 2\right) \times\left(F W H M_{\min } / 2\right)}=0.04 \mathrm{pc}\right)$.

The scaling factor of 15 corresponds to a spectral index $\alpha=3.0 \pm 0.4$ and we thus obtain an opacity spectral index $\beta=1.0 \pm 0.4$. The derived dust opacity index corresponds, taking the upper limit, to a "normal" interstellar dust material value. Nonetheless, if taking the lower limit, the dust opacity index decreases to a value that is usually found toward disks (e.g., Beckwith \& Sargent 1991; Natta et al. 2004), which has been ascribed to grain growth. Alternatively, the presence of winds/outflows (see Sect. 4.2) could mimic a low value of $\beta$ (Beuther et al. 2007). Here we adopt a $\beta=1.6$ in the rest of the paper.

\subsection{PdBI: molecular emission}

Spectroscopic parameters of the detected $\mathrm{C}^{34} \mathrm{~S} 2 \rightarrow 1$ and $\mathrm{CH}_{3} \mathrm{OH} 2_{k} \rightarrow 1_{k}$ and $5_{k} \rightarrow 4_{k}$ transitions toward G11.11P1 are listed in Table 3 . The interferometric integrated intensity maps, overlaid on their corresponding continuum emission, along with sample spectra are presented in Figs. 3-5.

\subsection{1. $\mathrm{C}^{34} \mathrm{~S}$ emission}

The integrated intensity map of the $\mathrm{C}^{34} \mathrm{~S} 2 \rightarrow 1$ line, is shown in the top panel of Fig. 3. This emission is spatially coincident
Table 3. Molecular parameters of detected line transitions.

\begin{tabular}{llrrr}
\hline \hline Molecule $^{a}$ & Transition & $\begin{array}{r}\text { Frequency } \\
(\mathrm{MHz})\end{array}$ & $\begin{array}{r}E_{\mathrm{u}} / k \\
(\mathrm{~K})\end{array}$ & $\begin{array}{r}\mu^{2} S \\
\left(\mathrm{D}^{2}\right)\end{array}$ \\
\hline $\mathrm{C}^{34} \mathrm{~S}$ & $2 \rightarrow 1$ & 96412.9495 & 6.94 & 7.6678 \\
$\mathrm{CH}_{3} \mathrm{OH}$ & $2_{-1} \rightarrow 1_{-1} E$ & 96739.3620 & 12.55 & 1.2134 \\
& $2_{0} \rightarrow 1_{0} A$ & 96741.3750 & 6.97 & 1.6170 \\
& $2_{0} \rightarrow 1_{0} E$ & 96744.5500 & 20.10 & 1.6166 \\
& $2_{1} \rightarrow 1_{1} E$ & 96755.5110 & 28.03 & 1.2442 \\
& $5_{0} \rightarrow 4_{0} E$ & 241700.2190 & 47.96 & 4.0402 \\
& $5_{-1} \rightarrow 4_{-1} E$ & 241767.2240 & 40.41 & 3.8824 \\
& $5_{0} \rightarrow 4_{0} A$ & 241791.4310 & 34.83 & 4.0430 \\
& $5_{ \pm 3} \rightarrow 4_{ \pm 3} A$ & 241832.9100 & 84.68 & 2.5775 \\
& $5_{1} \rightarrow 4_{1} E$ & 241879.0730 & 55.91 & 3.9802 \\
& $5_{-2} \rightarrow 4_{-2} E$ & $241904.1520^{b}$ & 60.76 & 3.3986 \\
& $5_{2} \rightarrow 4_{2} E$ & $241904.6450^{b}$ & 57.11 & 3.3558 \\
\hline
\end{tabular}

Notes. ${ }^{(a)}$ Rest frequencies, upper level energies and $\mu^{2} S$ from the Cologne Database for Molecular Spectroscopy (CDMS; Müller et al. 2001, 2005) as of December 2010. ${ }^{(b)}$ Blend of lines.
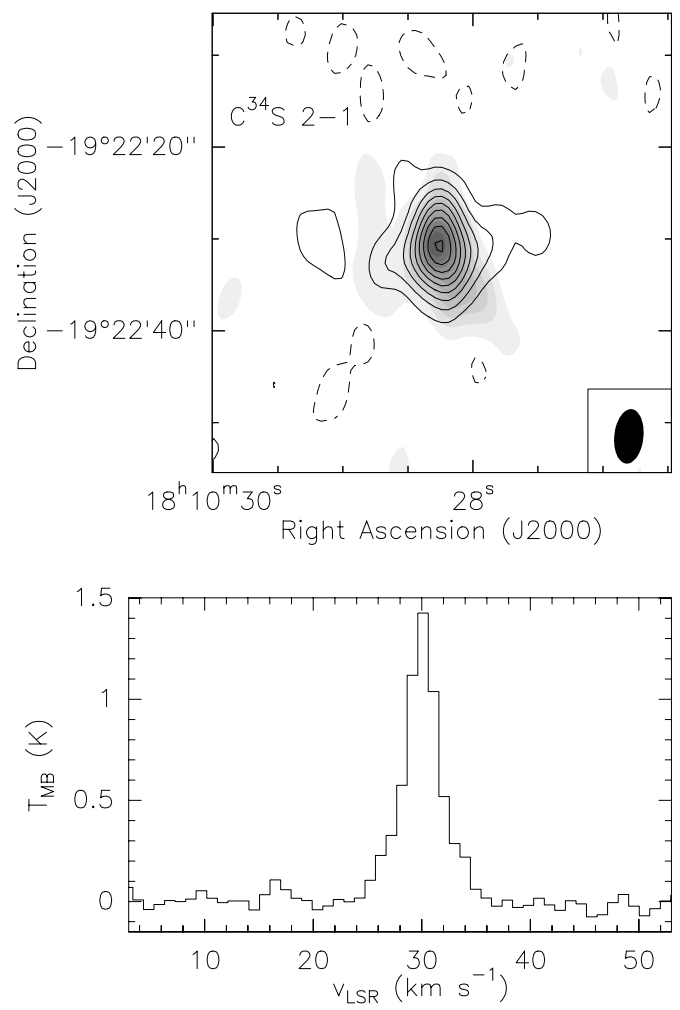

Fig. 3. Top: contour image of the emission integrated under the $\mathrm{C}^{34} \mathrm{~S} 2 \rightarrow 1$ line, in the velocity range from 22.4 to $37.9 \mathrm{~km} \mathrm{~s}^{-1}$, overlaid on the grey-scale $3.1 \mathrm{~mm}$ continuum emission of G11.11P1. First contour and contour spacing are $0.09 \mathrm{Jy}_{\text {beam }}^{-1} \mathrm{~km} \mathrm{~s}^{-1}(3 \sigma)$, the dashed contours show the negative emission $(-3 \sigma)$. The synthesized beam $\left(66^{\prime} 0 \times 33^{\prime \prime} 2 ; \mathrm{PA}=176^{\circ}\right)$ is shown in the lower right corner. Bottom: spectrum of $\mathrm{C}^{34} \mathrm{~S} 2 \rightarrow 1$ toward the peak position on the integrated intensity map at RA $=18^{\mathrm{h}} 10^{\mathrm{m}} 28^{\mathrm{s}} \cdot 24$, Dec $=-19^{\circ} 22^{\prime} 30^{\prime} \cdot 5(\mathrm{~J} 2000)$.

with the $3 \mathrm{~mm}$ continuum; moreover, some weaker emission (at $3 \sigma$ level) extends along the E-W direction. Observed parameters toward the peak position in the integrated intensity map are presented in Table 4.

Virial mass. The virial mass of a core with a power-law density distribution is $M_{\mathrm{vir}}=k_{1} \sigma_{\mathrm{v}}^{2} R / G$ (MacLaren et al. 1988), where 

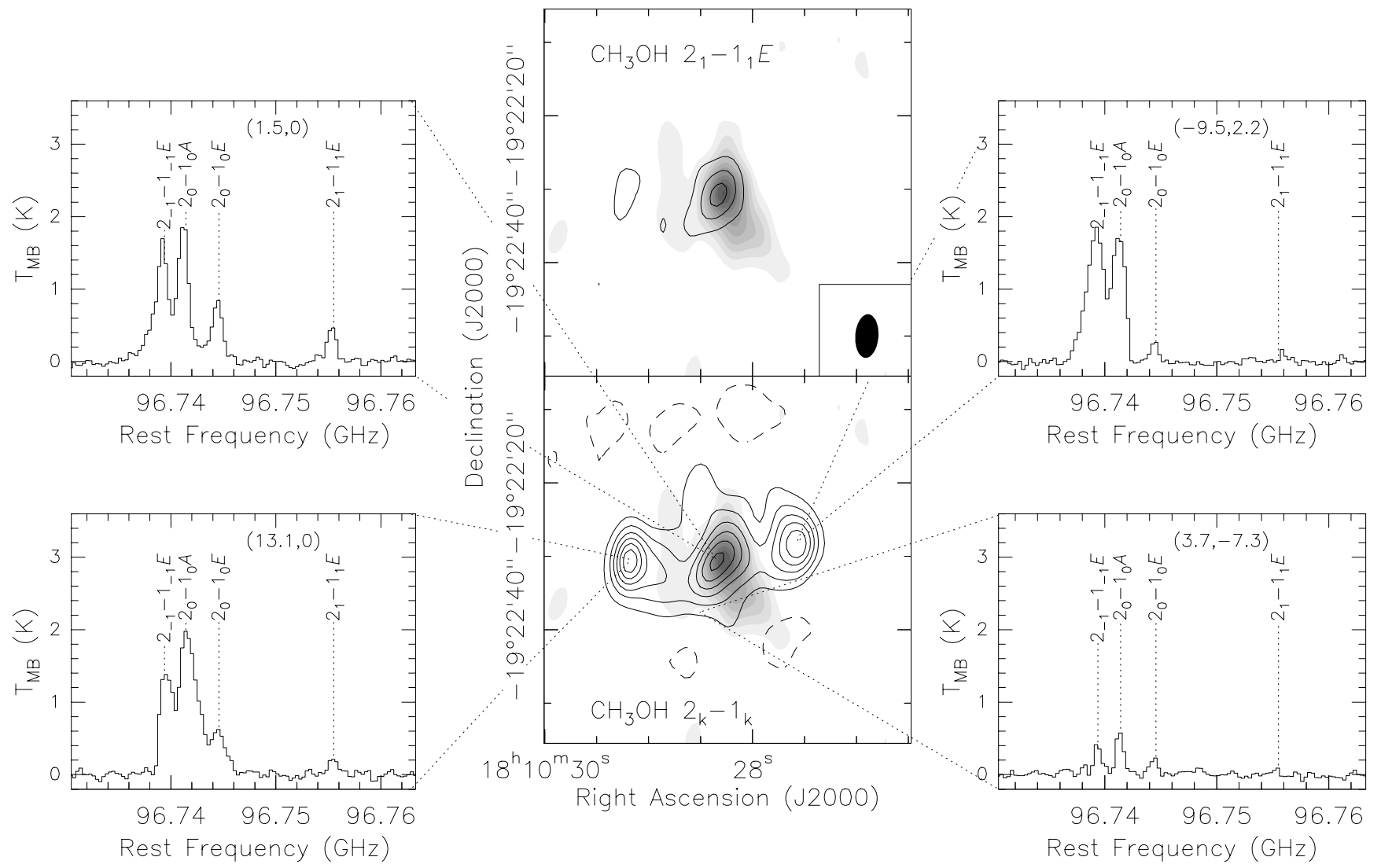

Fig. 4. In greyscale, the $3.1 \mathrm{~mm}$ continuum emission of G11.11P1. Top middle: contour image of the $\mathrm{CH}_{3} \mathrm{OH}$ emission integrated under the line $2_{1} \rightarrow 1_{1} E$. First contour and contour spacing are $0.66 \mathrm{Jy} \mathrm{beam}^{-1} \mathrm{~km} \mathrm{~s}^{-1}(3 \sigma)$. The synthesized beam $\left(6^{\prime \prime} 0 \times 33^{\prime \prime} 2 ; \mathrm{PA}=174^{\circ}\right)$ is shown in the lower right corner. Bottom middle: contour image of the $\mathrm{CH}_{3} \mathrm{OH}$ emission integrated under the $2_{-1} \rightarrow 1_{-1} E, 2_{0} \rightarrow 1_{0} A$, and $2_{0} \rightarrow 1_{0} E$ transition lines. First contour and contour spacing are $0.45 \mathrm{Jy} \mathrm{beam}^{-1} \mathrm{~km} \mathrm{~s}^{-1}(3 \sigma)$. The dashed contours show the negative emission $(-3 \sigma)$. We also show spectra at four different positions; positions are given in each inset in parenthesis in units of arcsec.

Table 4. Observed molecular line parameters from Gaussian fits.

\begin{tabular}{|c|c|c|c|c|c|}
\hline Molecule & Transition & $\begin{array}{c}v_{\mathrm{LSR}} \\
\left(\mathrm{km} \mathrm{s}^{-1}\right)\end{array}$ & $\begin{array}{l}T_{\mathrm{MB}} \\
(\mathrm{K})\end{array}$ & $\begin{array}{c}\Delta V(F W H M) \\
\left(\mathrm{km} \mathrm{s}^{-1}\right)\end{array}$ & $\begin{array}{c}\int_{\left(\mathrm{K} \mathrm{km} \mathrm{s}^{-1}\right)} T_{\mathrm{MB}} \mathrm{d} V \\
\end{array}$ \\
\hline \multicolumn{6}{|c|}{ Offset $\left(0^{\prime \prime}, 0^{\prime \prime}\right)$} \\
\hline $\mathrm{C}^{34} \mathrm{~S}$ & $2 \rightarrow 1$ & $29.90(0.04)$ & $1.31(0.04)$ & $4.1(0.1)$ & $5.6(0.1)$ \\
\hline \multicolumn{6}{|c|}{ Offset $\left(1^{\prime \prime} 5,0^{\prime \prime}\right)$} \\
\hline \multirow[t]{8}{*}{$\mathrm{CH}_{3} \mathrm{OH}$} & $2_{-1} \rightarrow 1_{-1} E$ & $29.85(0.05)$ & $1.49(0.04)$ & $5.1(0.2)$ & $8.0(0.2)$ \\
\hline & $2_{0} \rightarrow 1_{0} A$ & $29.72(0.04)$ & $1.89(0.04)$ & $3.6(0.1)$ & $7.3(0.2)$ \\
\hline & $2_{0} \rightarrow 1_{0} E$ & $29.87(0.09)$ & $0.78(0.04)$ & $4.4(0.2)$ & $3.6(0.1)$ \\
\hline & $2_{1} \rightarrow 1_{1} E$ & $29.79(0.01)$ & $0.49(0.04)$ & $3.1(0.2)$ & $1.6(0.1)$ \\
\hline & $5_{0} \rightarrow 4_{0} E$ & $29.71(0.20)$ & $0.70(0.04)$ & $4.1(0.5)$ & $3.1(0.3)$ \\
\hline & $5_{-1} \rightarrow 4_{-1} E$ & $29.92(0.09)$ & $1.48(0.04)$ & $4.1(0.2)$ & $6.5(0.3)$ \\
\hline & $5_{0} \rightarrow 4_{0} A$ & $30.00(0.05)$ & $1.80(0.04)$ & $4.3(0.2)$ & $8.3(0.3)$ \\
\hline & $5_{1} \rightarrow 4_{1} E$ & $29.78(0.41)$ & $0.33(0.04)$ & $3.9(1.3)$ & $1.4(0.3)$ \\
\hline
\end{tabular}

Notes. $\mathrm{CH}_{3} \mathrm{OH} 2_{k} \rightarrow 1_{k}$ and $5_{k} \rightarrow 4_{k}$ line parameters listed in this table were used in the rotational diagram method described in the text. The $\mathrm{CH}_{3} \mathrm{OH} 5_{k} \rightarrow 4_{k}$ transitions have been convolved to the $2_{k} \rightarrow 1_{k}$ resolution.

$k_{1}=(5-2 p) /(3-p), \sigma_{\mathrm{v}}$ is the three-dimensional root-meansquare velocity related to the $F W H M$ line width, $\Delta V$, through $\sigma_{\mathrm{v}}^{2}=(3 / 8 \ln 2) \Delta V^{2}, R$ is the core radius, and $G$ is the gravitational constant. For $p=1.6$ (see Sect. 3.1.3), the virial mass can be expressed as

$M_{\mathrm{vir}} \simeq 161 \times\left(\frac{R}{\mathrm{pc}}\right)\left(\frac{\Delta V}{\mathrm{~km} \mathrm{~s}^{-1}}\right)^{2} M_{\odot}$.
A virial mass of $\sim 135 M_{\odot}$ is calculated when using $\Delta V=$ $4.1 \mathrm{~km} \mathrm{~s}^{-1}$ (see Table 4) of the $\mathrm{C}^{34} \mathrm{~S}$ optically thin line and $R=0.05 \mathrm{pc}$, which corresponds to the geometric mean of the deconvolved linear size.

Virial parameter. The virial parameter (Bertoldi \& McKee 1992) for a spherical cloud is defined as $\alpha_{\mathrm{vir}} \equiv M_{\mathrm{vir}} / M_{\mathrm{tot}}$, 

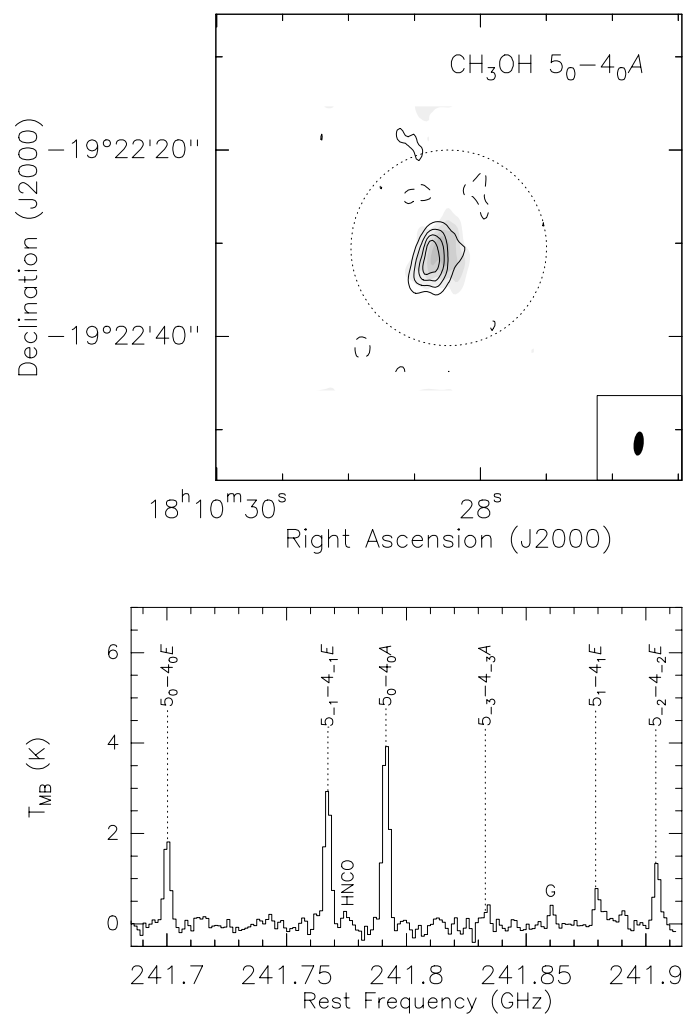

Fig. 5. Top: contour image of the emission integrated under the $5_{0} \rightarrow 4_{0} A$ transition line, overlaid on the greyscale $1.2 \mathrm{~mm}$ continuum emission of G11.11P1. First contour and contour spacing are $0.16 \mathrm{Jy}$ beam $^{-1} \mathrm{~km} \mathrm{~s}^{-1}(3 \sigma)$; the dashed contours show the negative emission $(-3 \sigma)$. The synthesized beam $\left(2^{\prime \prime} \cdot 6 \times 1^{\prime \prime}\right.$. 1 ; PA $\left.=174^{\circ}\right)$ is shown in the lower right corner. The dotted circle indicates the interferometer primary beam $\left(21^{\prime \prime}\right)$ at this frequency. Bottom: spectrum of $\mathrm{CH}_{3} \mathrm{OH} 5_{k} \rightarrow 4_{k}$ toward the position: RA $=18^{\mathrm{h}} 10^{\mathrm{m}} 28.40$, $\operatorname{Dec}=-19^{\circ} 22^{\prime} 31^{\prime \prime} .9(\mathrm{~J} 2000)$. The HNCO $11_{0,11} \rightarrow 10_{0,10}$ line (at $241774.0320 \mathrm{MHz}$ with $E_{\mathrm{u}} / k=69.67 \mathrm{~K}$ and $\mu^{2} S=27.4590 \mathrm{D}^{2}$ ) is shown as well. The " $G$ " shows an artifact produced in the central channels of a correlator unit by the Gibbs effect.

where $M_{\text {tot }}$ is the total mass. If we assume that $M_{\text {tot }}=M_{\mathrm{g}}=$ $37 M_{\odot}$, then $\alpha_{\text {vir }}=3.6$.

We note that the $\mathrm{C}^{34} \mathrm{~S}$ line width could be affected by unbound motions, e.g., outflows, partially broadening the line and thereby significantly increasing the virial mass estimate, in addition to the virial parameter.

Jeans mass. Following Stahler \& Palla (2005), we can calculate the Jeans mass

$M_{\mathrm{J}}=\frac{m a^{3}}{\rho^{1 / 2} G^{3 / 2}} \simeq 1.0 \times\left(\frac{T}{10 \mathrm{~K}}\right)^{3 / 2}\left(\frac{n}{10^{4} \mathrm{~cm}^{-3}}\right)^{-1 / 2} M_{\odot}$,

where $a$ is the isothermal sound speed and $\rho$ is the mass density. For $n=7 \times 10^{5} \mathrm{~cm}^{-3}$ (see Sect. 3.1.3) and $T=60 \mathrm{~K}$, we obtain a Jeans mass of $\sim 1.8 M_{\odot}$. We find a gas mass $\left(M_{\mathrm{g}}\right)$ to the Jeans mass $\left(M_{\mathrm{J}}\right)$ ratio larger than unity; other interferometric studies (Rathborne et al. 2008; Zhang et al. 2009) toward IRDCs also find that $M_{\mathrm{g}} / M_{\mathrm{J}}>1$.

From our interferometric observations at $1 \mathrm{~mm}$, and taking a flux density equal to the $3 \sigma$ detection level, we derive a mass sensitivity limit of $0.4 M_{\odot}$. Comparing this value with the Jeans mass, we note that our mass sensitivity limit is good enough to detect fragments of about the Jeans mass.

\subsection{2. $\mathrm{CH}_{3} \mathrm{OH}$ emission}

Figure 4 shows the integrated intensity map of the $\mathrm{CH}_{3} \mathrm{OH} 2_{k} \rightarrow$ $1_{k}$ quartet of lines (bottom middle). The emission exhibits three maxima: one is spatially coincident with the 1 and $3 \mathrm{~mm}$ continuum emission that peaks at $\left(1^{\prime \prime} .5,0^{\prime \prime}\right)$, and the other two are located at $\left(13^{\prime \prime} 1,0^{\prime \prime}\right)$ and $\left(-9 . ' 5,22^{\prime \prime} 2\right)$. We plot sample spectra at four different positions, including the three maxima and one position on the envelope $\left(33^{\prime \prime} 7,-7{ }^{\prime} \cdot 3\right)$, to compare the line profiles, absolute line strengths, and the relative strengths among lines. The high excitation $\mathrm{CH}_{3} \mathrm{OH} 2_{1} \rightarrow 1_{1} E$ transition that arises from a smaller region is also shown (top middle).

Line widths at and close to the three peak positions are on average $\sim 5 \mathrm{~km} \mathrm{~s}^{-1}$ with line profiles showing red- and/or blueshifted "wings", while in the outer parts of the envelope, e.g., at the $(3$ !' $7,-7$ '. 3$)$ position, the line width is narrower ( $\sim 2 \mathrm{~km} \mathrm{~s}^{-1}$; see spectra in Fig. 4).

In Fig. 5, we present the integrated intensity map of the strongest line $\mathrm{CH}_{3} \mathrm{OH} 5_{0} \rightarrow 4_{0} A$. The emission distribution is as extended as that of the $1 \mathrm{~mm}$ continuum and the $\mathrm{CH}_{3} \mathrm{OH} 2_{1} \rightarrow 1_{1} E$ emissions.

Local thermodynamic equilibrium (LTE) analysis. We derived the rotational temperatures and methanol column densities using the rotational diagram method (e.g., Cummins et al. 1986; Goldsmith \& Langer 1999). Briefly, for an optically thin line, the column density in the upper level $\left(N_{\mathrm{u}}\right)$ can be written as $N_{\mathrm{u}}=3 k W g_{\mathrm{u}} /\left(8 \pi^{3} v \mu^{2} S\right)$, where $k$ is the Boltzmann constant, $W$ is the integrated intensity of a line $\left(\int T_{\mathrm{MB}} \mathrm{d} V\right), g_{\mathrm{u}}$ the statistical weight, $v$ the frequency, $S$ the line strength, and $\mu$ the dipole moment. Under LTE conditions, the population of each level follows a Boltzmann law at the gas temperature $T$ by $N_{\mathrm{u}}=(N / Q) g_{\mathrm{u}} e^{-E_{\mathrm{u}} / k T}$, where $N$ is the total column density, $Q$ the partition function, and $E_{\mathrm{u}}$ the energy of the upper level. We then obtain

$\log \left(\frac{3 k W}{8 \pi^{3} v \mu^{2} S}\right)=\log \left(\frac{N}{Q}\right)-\frac{\log e}{T} \frac{E_{\mathrm{u}}}{k}$.

Using Eq. (4), we plot $\log \left(\frac{3 k W}{8 \pi^{3} v \mu^{2} S}\right)$ versus $E_{\mathrm{u}} / k$ (see Fig. 6) and perform a least squares fit for $T$ and $N$ taking into account both the $\mathrm{CH}_{3} \mathrm{OH} 2_{k} \rightarrow 1_{k}$ and $5_{k} \rightarrow 4_{k}$ bands. The partition function of $\mathrm{CH}_{3} \mathrm{OH}$ is $Q=2\left[\frac{\pi(k T)^{3}}{h^{3} A B C}\right]^{1 / 2}$ (Turner 1991), where $h$ is the Planck constant and $A, B$, and $C$ are the rotation constants (CDMS; Müller et al. 2001, 2005). A $Q=1.23 T^{1.5}$ relation was used in the calculations. The $\mathrm{CH}_{3} \mathrm{OH} 5_{k} \rightarrow 4_{k}$ data were smoothed to the resolution of the $2_{k} \rightarrow 1_{k}$ data. The $\mathrm{CH}_{3} \mathrm{OH}$ transition lines that were used in this fit are listed in Table 4.

To estimate the $\mathrm{CH}_{3} \mathrm{OH}$ fractional abundance (relative to $\mathrm{H}_{2}$ ), $X_{\mathrm{CH}_{3} \mathrm{OH}}$, we calculate the beam-averaged $\mathrm{H}_{2}$ column density, $N_{\mathrm{H}_{2}}$, following the expression

$N_{\mathrm{H}_{2}}=\frac{I_{v}^{\text {peak }} R_{\mathrm{gd}}}{\kappa_{v} B_{v}\left(T_{\mathrm{d}}\right) \Omega \mu_{\mathrm{H}_{2}} m_{\mathrm{H}}} \mathrm{cm}^{-2}$,

where $I_{v}^{\text {peak }}$ is the peak intensity in Jy/beam, $\mu_{\mathrm{H}_{2}}=2.8$ (Kauffmann et al. 2008) is the molecular weight per hydrogen molecule, and $\Omega=\left(\pi \theta_{\min } \theta_{\text {maj }}\right) /(4 \ln 2)$ is the beam solid angle of an elliptical Gaussian beam with minor and major axes $\theta_{\min }$ and $\theta_{\text {maj }}$, respectively.

We obtained a rotational temperature of $15 \mathrm{~K}$ and a $\mathrm{CH}_{3} \mathrm{OH}$ column density, $N_{\mathrm{CH}_{3} \mathrm{OH}}$, of $1.2 \times 10^{15} \mathrm{~cm}^{-2}$ toward the $\left(1^{\prime \prime} .5,0^{\prime \prime}\right)$ peak position. Since the lines are likely sub-thermally excited, we take a dust temperature $T_{\mathrm{d}}=60 \mathrm{~K}$ (see LVG analysis) 
L. Gómez et al.: Observations of methanol in the IRDC core G11.11-0.12P1

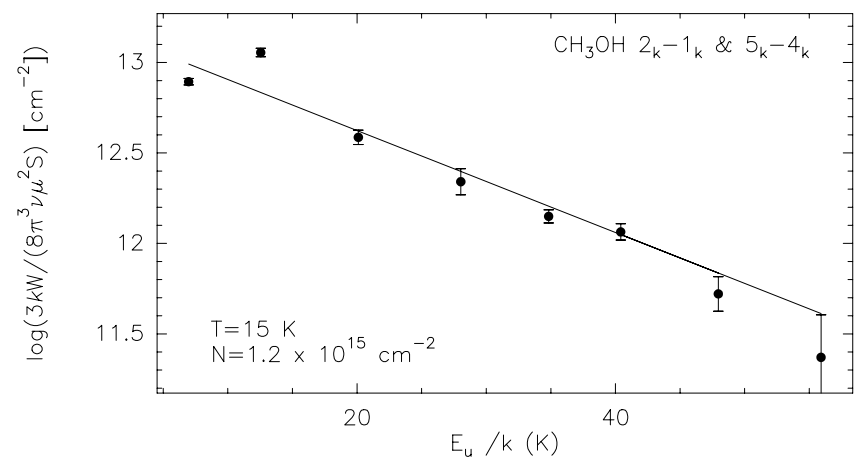

Fig. 6. Rotational diagram of $\mathrm{CH}_{3} \mathrm{OH}(2-1)$ and (5-4) transition lines toward the $\left(11^{\prime \prime} 5,0^{\prime \prime}\right)$ position. The straight line is a least squares fit to the data corresponding to the rotational temperature and total column density indicated in the bottom left. Error bars are for $1 \sigma$ uncertainties.

and obtain a $N_{\mathrm{H}_{2}}=4.6 \times 10^{22} \mathrm{~cm}^{-2}$ (from our $1 \mathrm{~mm}$ continuum data convolved to the $3 \mathrm{~mm}$ data) and a $X_{\mathrm{CH}_{3} \mathrm{OH}}$ of $2.6 \times 10^{-8}$.

At the $\left(13^{\prime \prime} 1,0^{\prime \prime}\right)$ position, we obtain $X_{\mathrm{CH}_{3} \mathrm{OH}}=3.7 \times 10^{-9}$, when assuming that $N_{\mathrm{CH}_{3} \mathrm{OH}}=2.9 \times 10^{14} \mathrm{~cm}^{-2}$ and $N_{\mathrm{H}_{2}}=7.7 \times$ $10^{22} \mathrm{~cm}^{-2}$. A rotational temperature of $13 \mathrm{~K}$ was calculated. At the $\left(-9 . ' 5,2\right.$ '. 2 ) position, the values for the $X_{\mathrm{CH}_{3} \mathrm{OH}}, N_{\mathrm{CH}_{3} \mathrm{OH}}$, and $N_{\mathrm{H}_{2}}$ are $5.6 \times 10^{-9}, 3.8 \times 10^{14} \mathrm{~cm}^{-2}$, and $6.8 \times 10^{22} \mathrm{~cm}^{-2}$, respectively. For the $(-9.5,2$.'2) peak, we fixed $T=15 \mathrm{~K}$ because of the low signal-to-noise ratio of one of the three lines used in the fit. For both peaks at $\left(13^{\prime \prime} 1,0^{\prime \prime}\right)$ and $\left(-9 .{ }^{\prime} 5,2\right.$.'2), $N_{\mathrm{CH}_{3} \mathrm{OH}}$ wa calculated from the $3 \mathrm{~mm}$ interferometric map only and $N_{\mathrm{H}_{2}}$ from the SCUBA $850 \mu \mathrm{m}$ dust continuum; the interferometric observations were convolved to the SCUBA beam size $\left(14^{\prime \prime}\right)$.

Large velocity gradient (LVG) analysis. Leurini et al. (2004) found several ratios, from some $\mathrm{CH}_{3} \mathrm{OH}-E$ transitions, to be calibration-independent tracers of density in the 1 and $3 \mathrm{~mm}$ bands. We used their LVG calculations and plotted the integrated intensity line ratios $5_{0} \rightarrow 4_{0} / 5_{-1} \rightarrow 4_{-1}, 5_{1} \rightarrow 4_{1} / 5_{-1} \rightarrow 4_{-1}$, and $5_{1} \rightarrow 4_{1} / 5_{0} \rightarrow 4_{0}$, in a temperature-density $(T-n)$ plane. In Fig. 7 , the observed ratios, toward the $\left(1^{\prime \prime} .5,0^{\prime \prime}\right)$ position, are represented by the black dash-dotted line, blue solid line, and pink dashed line. The $2_{k} \rightarrow 1_{k}$ quartet of lines in the $3 \mathrm{~mm}$ band were not used in the analysis because they are blended.

Given the results for $\mathrm{CH}_{3} \mathrm{OH}$ column densities in the LTE analysis, the explored LVG models for the excitation of methanol run for temperatures in the range of $10-200 \mathrm{~K}$, for densities $10^{5}-10^{8} \mathrm{~cm}^{-3}$, and for two $\mathrm{CH}_{3} \mathrm{OH}$ column densities per line width $10^{14}$ and $10^{15} \mathrm{~cm}^{-2} /\left(\mathrm{km} \mathrm{s}^{-1}\right)$.

For an average line width of $4 \mathrm{~km} \mathrm{~s}^{-1}$, this corresponds to $N_{\mathrm{CH}_{3} \mathrm{OH}-\mathrm{E}}=4 \times 10^{14} \mathrm{~cm}^{-2}$ and $4 \times 10^{15} \mathrm{~cm}^{-2}$, respectively. Depending on the column density, two possible solutions are found. At $N_{\mathrm{CH}_{3} \mathrm{OH}-\mathrm{E}}=4 \times 10^{14} \mathrm{~cm}^{-2}$, the three integrated intensity line ratios intercept at $T=17 \mathrm{~K}$ and $n=10^{7} \mathrm{~cm}^{-3}$, whereas at $N_{\mathrm{CH}_{3} \mathrm{OH}-\mathrm{E}}=4 \times 10^{15} \mathrm{~cm}^{-2}$, the interception is at $T=60 \mathrm{~K}$ and $n=2 \times 10^{6} \mathrm{~cm}^{-3}$ (see Fig. 7). The methanol column density inferred in this case is consistent with the LTE analysis to within a factor of three, but the intensities in this model are one order of magnitude higher than the observed line intensities. In other words, to fit the observations with the $N_{\mathrm{CH}_{3} \mathrm{OH}-\mathrm{E}}=4 \times$ $10^{15} \mathrm{~cm}^{-2}$ model, we need a beam filling factor of 0.1 resulting in a more compact source (with a size of 0.5 corresponding to $0.009 \mathrm{pc}$ or $1800 \mathrm{AU})$, even smaller than the one modeled by Pillai et al. (2006a) and Leurini et al. (2007b).
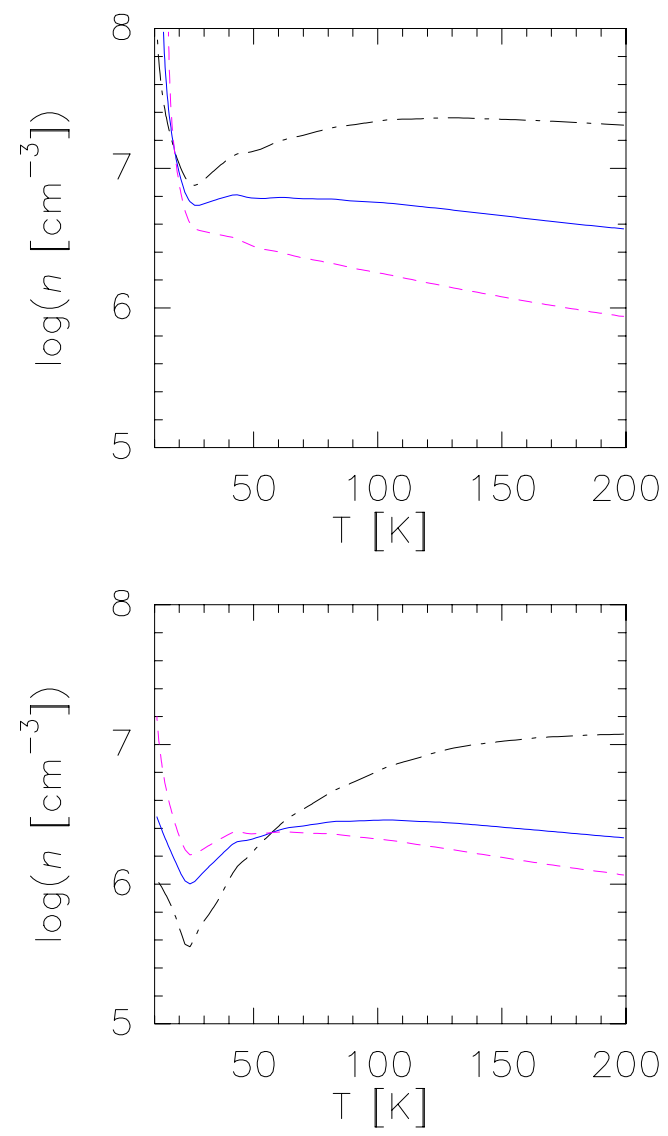

Fig. 7. Results of statistical equilibrium calculations for $\mathrm{CH}_{3} \mathrm{OH}-E$. The $5_{0} \rightarrow 4_{0} / 5_{-1} \rightarrow 4_{-1}$ (black dash-dotted line), $5_{1} \rightarrow 4_{1} / 5_{-1} \rightarrow 4_{-1}$ (blue solid line), and $5_{1} \rightarrow 4_{1} / 5_{0} \rightarrow 4_{0}$ (pink dashed line) observed integrated intensity line ratios toward the $\left(11^{\prime \prime} 5,0^{\prime \prime}\right)$ position on a logarithmic scale are shown as function of $\mathrm{H}_{2}$ density and temperature at $N_{\mathrm{CH}_{3} \mathrm{OH}} / \Delta V=10^{14} \mathrm{~cm}^{-2} /\left(\mathrm{km} \mathrm{s}^{-1}\right)$ (top) and $N_{\mathrm{CH}_{3} \mathrm{OH}} / \Delta V=$ $10^{15} \mathrm{~cm}^{-2} /\left(\mathrm{km} \mathrm{s}^{-1}\right)$ (bottom).

To gain more insight into the possible $T-n$ degeneracy, in Fig. 8, we show $n$ versus $N_{\mathrm{CH}_{3} \mathrm{OH}}$ plots, at different temperatures $(10-70 \mathrm{~K})$, where the color-coding for the ratios (along with their $1 \sigma$ values) are the same as in Fig. 7; in addition, we show in each panel the peak main beam brightness temperature of the $5_{0} \rightarrow 4_{0} E$ transition (green dotted line). The parameters obtained in the regions in the left side of the green dotted line imply beam filling factors greater than 1 , which is not possible. In each panel, the areas of interest are those that lie in the right side of the green line and, at the same time, are delimited by the integrated intensity ratios (taking into account their $1 \sigma$ values). We see that, for $T$ in the range $30-70 \mathrm{~K}$, the $n$ remains constant up to $N_{\mathrm{CH}_{3} \mathrm{OH}} \sim 4 \times 10^{14} \mathrm{~cm}^{-2}$, while for very low $T(=10 \mathrm{~K})$ there is no solution.

Given our density estimate from the dust continuum, we regard the low $T$, high $n$ solutions with a large filling factor as unlikely. Therefore, although we cannot strongly constrain the physical parameters of the gas emitting methanol from the LVG analysis because of the limited number of observed transitions, we can exclude the low temperature solution and place a lower limit on the kinetic temperature of the gas of $30 \mathrm{~K}$. This result agrees with the detection of methanol maser from this region and with a previous measurement of $T=60 \mathrm{~K}$ from $\mathrm{NH}_{3}$ observations. Therefore, we adopt $T=60 \mathrm{~K}$ as the kinetic temperature at the peak of the continuum emission. 


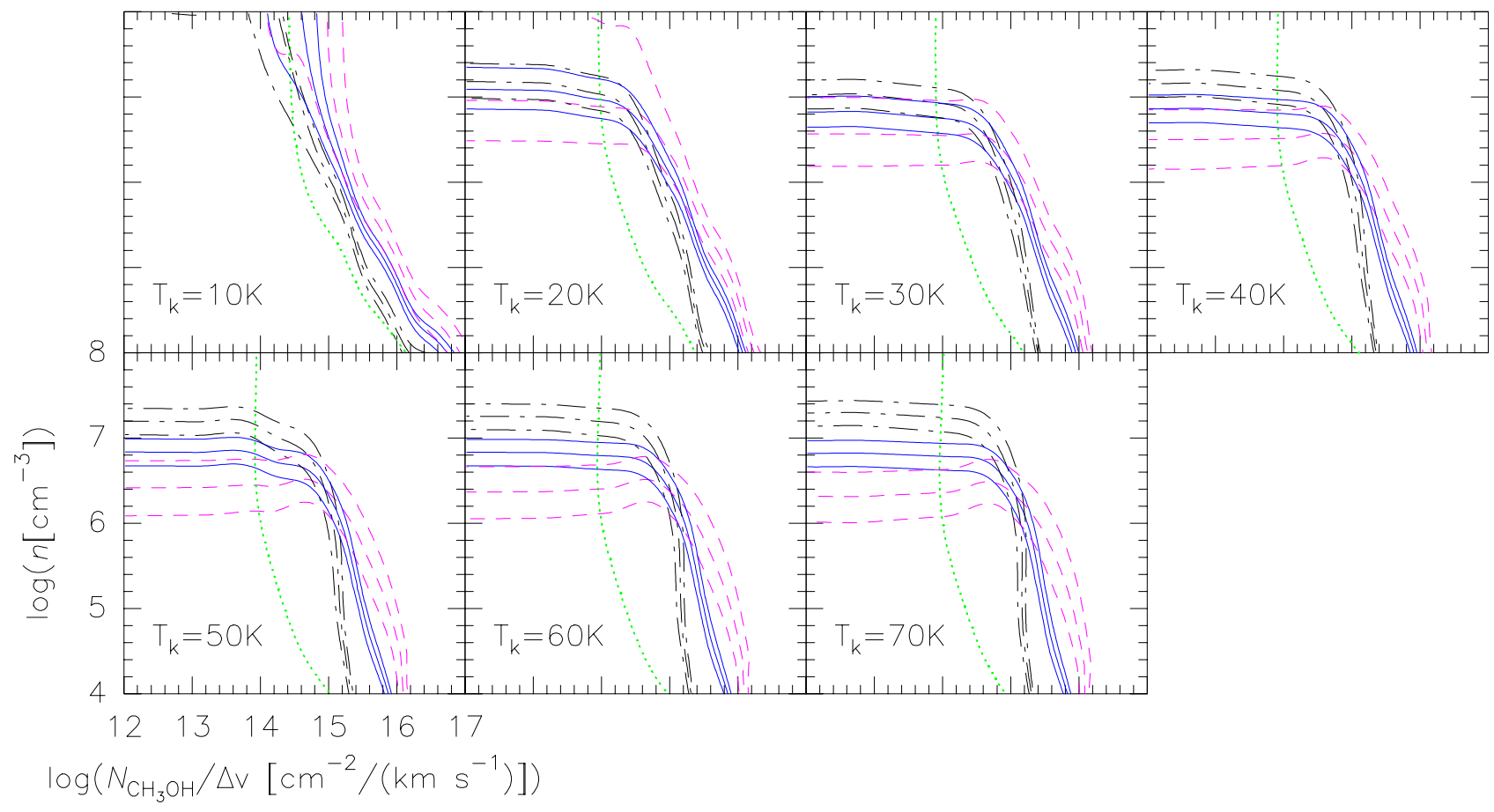

Fig. 8. Results of statistical equilibrium calculations for $\mathrm{CH}_{3} \mathrm{OH}-E$. Observed integrated intensity line ratios are color-coded as in Fig. 7 and plotted on a logarithmic scale (along with their $1 \sigma$ values) as a function of $\mathrm{H}_{2}$ density and $N_{\mathrm{CH}_{3} \mathrm{OH}} / \Delta V$ at different temperatures (10-70 K). The green dotted line represents the peak main brightness temperature of the $5_{0} \rightarrow 4_{0} E$ transition.

\section{3. $\mathrm{PdBI}+30 \mathrm{~m}$ : extended $\mathrm{CH}_{3} \mathrm{OH}$ emission}

A comparison of the interferometric data with the single-dish observations shows that only $\sim 30 \%$ of the $\mathrm{CH}_{3} \mathrm{OH} 2_{k} \rightarrow 1_{k}$ measured with the $30 \mathrm{~m}$ telescope is imaged in the PdBI observations. The combined PdBI and $30 \mathrm{~m}$ telescope data recover almost $100 \%$ of the flux at the central $\left(1^{\prime \prime} 5,0^{\prime \prime}\right)$ position. In Fig. 9, we show the resultant map after combining the $\mathrm{CH}_{3} \mathrm{OH} 2_{k} \rightarrow 1_{k}$ single-dish observations with the corresponding PdBI data. The methanol emission is already extended in the interferometric observations (mapping angular scales as large as $322^{\prime \prime} 5$ ) and confirmed to be so in the resultant merged map with the single-dish data. We note that the emission extends over a $1 \mathrm{pc}$ scale in the merged map (see Fig. 9). Similarly, silicon monoxide $(\mathrm{SiO})$ was found to be widespread over a 2 pc scale in the IRDC G035.39-00.33 (Jiménez-Serra et al. 2010). The $2_{1} \rightarrow$ $1_{1} E$ transition was not detected in the single-dish observations, which is expected since this emission does not appear to be extended in our interferometric data.

As for the $1 \mathrm{~mm}$ data, the missing flux in the interferometric observations is $\sim 70 \%$. In the $1 \mathrm{~mm}$ single-dish observations, two of the strongest transitions showed up, namely the $5_{-1} \rightarrow 4_{-1} E$ and the $5_{0} \rightarrow 4_{0} A$ lines. Both lines are above $3 \sigma$ noise level at 6 of 144 observed positions.

How is the derivation of column densities and rotational temperatures affected by missing flux? Using the combined data at $3 \mathrm{~mm}$ only, the slope, i.e., the rotational temperature, in the rotational diagram is unaffected, within the errors, while $\mathrm{CH}_{3} \mathrm{OH}$ column densities are higher by a factor of two.

\section{Discussion}

\subsection{Properties of G11.11P1 relative to those of other cores}

Despite our interferometric observations having sufficient sensitivity to resolve the Jeans mass $\left(1.8 M_{\odot}\right)$ at $1 \mathrm{~mm}$, the angular resolution $\left(22^{\prime \prime} 6 \times 11^{\prime \prime} 1\right)$ is smaller than but very close to the Jeans length of $33^{\prime \prime} 2(0.05 \mathrm{pc})$. Therefore, it is difficult to ascertain whether G11.11P1 is fragmenting into condensations (referred to as substructures within the cores) as is often found when zooming into these cores (e.g., Zhang et al. 2009; Bontemps et al. 2010). If G11.11P1 were not to fragment, this would mean that it has already accumulated most of its mass that will go into the final star even though $75 \%$ of the (dust) emission is filtered out by the interferometer. With higher angular resolution observations, it will be possible to determine whether either possibility is true.

The virial parameter of G11.11P1 obtained by Pillai et al. (2006a), from single-dish $\mathrm{NH}_{3}$ and dust continuum (at $870 \mu \mathrm{m}$ ) observations, is unity on the scale of $\sim 0.9 \mathrm{pc}$. Although, in the present work, we find that $M_{\text {vir }}$ is much higher than the $M_{\mathrm{g}}$ on the scale of $\sim 0.1 \mathrm{pc}$, which would indicate that gravity is not the dominant force (Bertoldi \& McKee 1992). We caution, however, that the high value of the virial parameter $\left(\alpha_{\mathrm{vir}}=3.6\right)$ could be due to the missing flux in the interferometric data and to the virial mass being estimated from the $\mathrm{C}^{34} \mathrm{~S}$ line width, whose broadening is likely to be in part produced by outflows. Pillai et al. (2006b) reported an increase in line widths from $1.29 \mathrm{~km} \mathrm{~s}^{-1}$ in the $\mathrm{NH}_{3}(1,1)$ line to $3.2 \mathrm{~km} \mathrm{~s}^{-1}$ in the $\mathrm{NH}_{3}(3,3)$ data. The increase in $\mathrm{NH}_{3}$ line width toward the higher $(J, K)$ transitions suggest that star forming activity has a strong influence on the line width on smaller scales. The broad line width in the $\mathrm{C}^{34} \mathrm{~S}$ from PdBI is consistent with that notion.

On the basis of the SCUBA $870 \mu \mathrm{m}$ column density, we estimated a surface density of $\Sigma=0.4 \mathrm{~g} \mathrm{~cm}^{-2}$ for G11.11P1, which is close to the thresholds of $0.7-1.5 \mathrm{~g} \mathrm{~cm}^{-2}$ (or $3320-7110 M_{\odot} \mathrm{pc}^{-2}$ ) required to form stars of $10-200 M_{\odot}$ (Krumholz \& McKee 2008). We note that these authors were interested in clouds where no massive stars have yet formed, while G11.11P1 shows already signposts of star formation activity. 


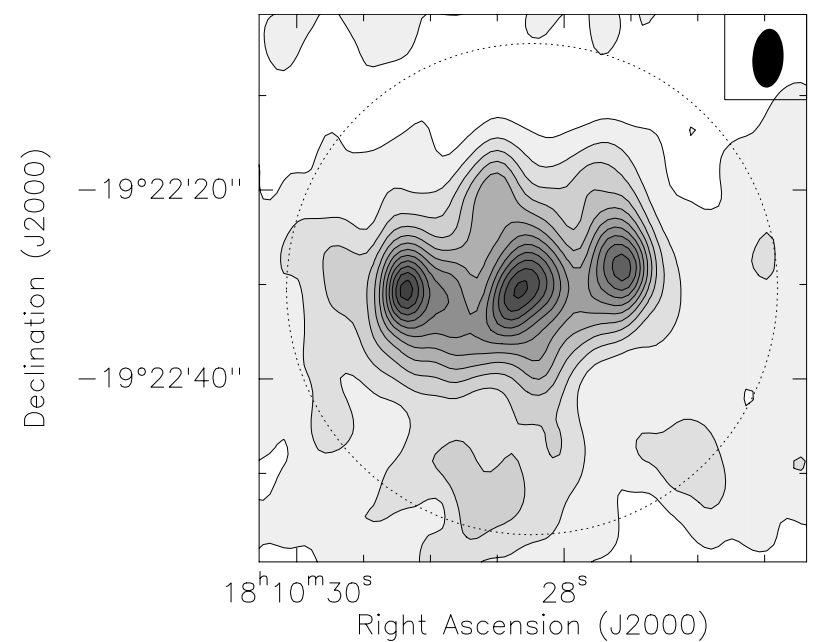

Fig. 9. $\mathrm{CH}_{3} \mathrm{OH} 2_{k} \rightarrow 1_{k} v_{t}=0$ integrated intensity map combining $\mathrm{PdBI}$ and $30 \mathrm{~m}$ data. The emission is integrated over the $2_{-1} \rightarrow 1_{-1} E$, $2_{0} \rightarrow 1_{0} A$, and $2_{0} \rightarrow 1_{0} E$ transitions. First contour and contour spacing are $0.39 \mathrm{Jy} \mathrm{beam}^{-1} \mathrm{~km} \mathrm{~s}^{-1}(3 \sigma)$, the dashed contours show the negative emission $(-3 \sigma)$. The synthesized beam $\left(6^{\prime \prime} \cdot 2 \times 3^{\prime \prime} \cdot 3\right.$; $\left.\mathrm{PA}=176^{\circ}\right)$ is shown in the upper right corner. The dotted circle indicates the interferometer primary beam $\left(52^{\prime \prime}\right)$.

We calculated a density profile power-law index of 1.6 from interferometric observations at 1 and $3 \mathrm{~mm}$; similar values, between 1.5 and 2, for cores in the IRDC G28.34+0.06, were reported by Zhang et al. (2009) also for embedded protostellar sources in the protocluster IRAS 05358+3543 (Beuther et al. 2007).

\subsection{The disk/outflow system?}

Pillai et al. (2006b) found a velocity gradient in the $\mathrm{CH}_{3} \mathrm{OH}$ maser emission and explained it as the maser spots being located in a Keplerian disk. Interestingly, the spread of the maser components is in the north-south direction, which is perpendicular to the $\mathrm{CH}_{3} \mathrm{OH} 2_{k} \rightarrow 1_{k}$ emission that we propose originates by the presence of an outflow (in the eastwest direction). The spread of the masing spots is translated into a radius of $\sim 450 \mathrm{AU}$ for a hypothetical disk in G11.11P1. Cesaroni et al. (2006) reported that candidate disks in high-mass protostars have masses in the range $0.2-40 M_{\odot}$ and radii of 500-10000 AU. Our interferometric data set is capable of imaging the largest disk structures, but not those inferred in Pillai et al. (2006b).

G11.11P1 was cataloged by Cyganowski et al. (2008) as a "possible" massive young stellar object (YSO) outflow candidate. This categorization was based on the angular extent of the extended excess $4.5 \mu \mathrm{m}$ emission, i.e., the extent of green emission in a three-color RGB image.

More evidence pointing to the presence of outflows comes from the non-Gaussian methanol line profiles (see Fig. 4 and Leurini et al. 2007b). In Fig. 10, we analyze the molecular emission in a position-velocity plot and note that there are "wings" of redshifted emission toward negative offsets and blueshifted emission toward positive offsets (see also Fig. 4). Moreover, we find a gradient of $\sim 4 \mathrm{~km} \mathrm{~s}^{-1}$, in the east-west direction, for the $\mathrm{CH}_{3} \mathrm{OH} 2_{k} \rightarrow 1_{k}$ emission and speculate that it is due to an outflow(s)-cloud interaction. The $\mathrm{C}^{34} \mathrm{~S} 2 \rightarrow 1$ and $\mathrm{CH}_{3} \mathrm{OH} 5_{k} \rightarrow$ $4_{k}$ lines are detected mainly toward the central peak/dust emission, and no velocity gradient is found.

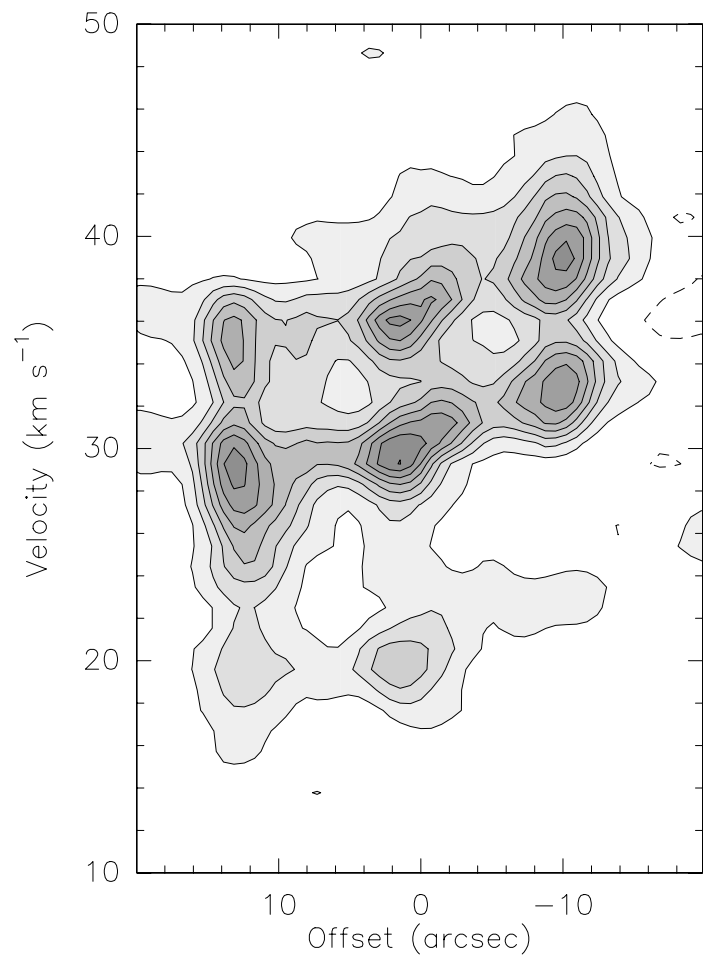

Fig. 10. Position-velocity plot of the methanol $2_{k} \rightarrow 1_{k}$ lines, cutting along the $\mathrm{PA}=96^{\circ}$ on the map shown in the middle bottom panel of Fig. 4 . We show the transitions: $2_{-1} \rightarrow 1_{-1} E, 2_{0} \rightarrow 1_{0} A$, and $2_{1} \rightarrow 1_{1} E$. The offset is measured in a positive direction toward east and from the pointing center of the observations. First contour is 0.018 in steps of $0.036 \mathrm{Jy} \mathrm{beam}^{-1}$, the dashed contours show the negative emission $\left(-0.018 \mathrm{Jy} \mathrm{beam}^{-1}\right)$.

Another possibility is that the $\mathrm{CH}_{3} \mathrm{OH} 2_{k} \rightarrow 1_{k}$ emission is actually tracing a toroid (see Cesaroni et al. 2007) as in the interferometric observations of $\mathrm{CH}_{3} \mathrm{OH} 6_{0} \rightarrow 5_{0}$ toward the IRDC 18223-3 (Fallscheer et al. 2009). However, the gradient of the maser spots is orthogonal and not aligned with the gradient of the methanol emission that we present in Fig. 10.

\section{3. $\mathrm{CH}_{3} \mathrm{OH}$ abundances}

Because methanol molecules can be sub-thermally excited (Bachiller et al. 1995), the temperatures derived from the rotational diagram are low $(\sim 15 \mathrm{~K})$, even at the central peak where masers have been detected. To overcome this problem we used the LVG method and found that a higher temperature is more reliable.

Purely gas-phase model calculations predict that $X_{\mathrm{CH}_{3} \mathrm{OH}}$ is on the order of $10^{-13}-10^{-10}$ (Garrod et al. 2006), which cannot reproduce the high values we report in this paper. Garrod et al. (2006) conclude that the production of methanol is carried out on the surfaces of dust grains followed by desorption into the gas. The $X_{\mathrm{CH}_{3} \mathrm{OH}}$ values in G11.P11 could then be produced by desorption of icy mantles on the dust grains.

As found in Sect. 3.2.2, the highest $X_{\mathrm{CH}_{3} \mathrm{OH}}$ in G11.11P1 is at the central position $\left(X_{\mathrm{CH}_{3} \mathrm{OH}} \sim 3 \times 10^{-8}\right)$, and is lower by more than one order of magnitude at the other two peaks, where $X_{\mathrm{CH}_{3} \mathrm{OH}} \sim 4-6 \times 10^{-9}$; the same trend was also found by the LVG-modeling of single-dish data (Leurini et al. 2007b). Our abundances are enhanced relative to dark cloud values $\left(X_{\mathrm{CH}_{3} \mathrm{OH}} \sim 10^{-10}-10^{-9}\right.$; Friberg et al. 1988), also supporting the 
idea that methanol in G11.11P1 is forming mainly through nonthermal desorption by the presence of outflow(s).

\section{Summary}

We have performed continuum and line observations with the PdBI toward the IRDC core G11.11P1. Our main results can be summarized as follows:

- The analysis of the mm continuum maps provide a very detailed physical structure of the core.

- Evidence that we have uncovered, i.e., "wings" in the $\mathrm{CH}_{3} \mathrm{OH} 2_{k} \rightarrow 1_{k}$ spectra, and a $\mathrm{CH}_{3} \mathrm{OH}$ abundance enhancement point to the presence of an outflow in the east-west direction.

- We have found a gradient of $\sim 4 \mathrm{~km} \mathrm{~s}^{-1}$ for the $\mathrm{CH}_{3} \mathrm{OH} 2_{k} \rightarrow$ $1_{k}$ emission, which we interpret as being produced by an outflow(s)-cloud interaction.

- Our fitting results have found an enhanced methanol fractional abundance $\left(\sim 3 \times 10^{-8}\right)$ at the central peak with respect to the other two maxima, where the methanol abundance is lower by about an order of magnitude $\left(\sim 4-6 \times 10^{-9}\right)$.

Acknowledgements. We acknowledge the IRAM staff at Pico Veleta and Plateau de Bure for carrying out the observations. L.G. thanks A. Castro-Carrizo and J. M. Winters for their help during the PdBI data reduction. We thank B. Parise for her evaluation of the manuscript and the referee for providing helpful comments and suggestions. L.G. was supported for this research through a stipend from the International Max Planck Research School (IMPRS) for Astronomy and Astrophysics at the Universities of Bonn and Cologne. T.P. acknowledges support from the Combined Array for Research in Millimeter-wave Astronomy (CARMA), which is supported by the National Science Foundation through grant AST 05-40399.

\section{References}

Aguirre, J. E., Ginsburg, A. G., Dunham, M. K., et al. 2011, ApJS, 192, 4 Bachiller, R., Liechti, S., Walmsley, C. M., \& Colomer, F. 1995, A\&A, 295, L51 Beckwith, S. V. W., \& Sargent, A. I. 1991, ApJ, 381, 250

Beckwith, S. V. W., Sargent, A. I., Chini, R. S., \& Guesten, R. 1990, AJ, 99, 924 Bertoldi, F., \& McKee, C. F. 1992, ApJ, 395, 140

Beuther, H., Leurini, S., Schilke, P., et al. 2007, A\&A, 466, 1065

Bontemps, S., Motte, F., Csengeri, T., \& Schneider, N. 2010, A\&A, 524, A18

Carey, S. J., Clark, F. O., Egan, M. P., et al. 1998, ApJ, 508, 721

Carey, S. J., Feldman, P. A., Redman, R. O., et al. 2000, ApJ, 543, L157
Cesaroni, R., Galli, D., Lodato, G., Walmsley, M., \& Zhang, Q. 2006, Nature, 444, 703

Cesaroni, R., Galli, D., Lodato, G., Walmsley, C. M., \& Zhang, Q. 2007, Protostars and Planets V, 197

Cummins, S. E., Linke, R. A., \& Thaddeus, P. 1986, ApJS, 60, 819

Cyganowski, C. J., Whitney, B. A., Holden, E., et al. 2008, AJ, 136, 2391

Egan, M. P., Shipman, R. F., Price, S. D., et al. 1998, ApJ, 494, L199

Fallscheer, C., Beuther, H., Zhang, Q., Keto, E., \& Sridharan, T. K. 2009, A\&A, 504,127

Friberg, P., Hjalmarson, A., Madden, S. C., \& Irvine, W. M. 1988, A\&A, 195, 281

Garay, G., Mardones, D., Rodríguez, L. F., Caselli, P., \& Bourke, T. L. 2002, ApJ, 567, 980

Garrod, R., Park, I. H., Caselli, P., \& Herbst, E. 2006, Chemical Evolution of the Universe, Faraday Discussions, 133, 51

Goldreich, P., \& Kwan, J. 1974, ApJ, 189, 441

Goldsmith, P. F., \& Langer, W. D. 1999, ApJ, 517, 209

Guilloteau, S., Delannoy, J., Downes, D., et al. 1992, A\&A, 262, 624

Henning, T., Linz, H., Krause, O., et al. 2010, A\&A, 518, L95

Hildebrand, R. H. 1983, QJRAS, 24, 267

Jiménez-Serra, I., Caselli, P., Tan, J. C., et al. 2010, MNRAS, 406, 187

Kauffmann, J., Bertoldi, F., Bourke, T. L., Evans, II, N. J., \& Lee, C. W. 2008, A\&A, 487, 993

Kristensen, L. E., van Dishoeck, E. F., van Kempen, T. A., et al. 2010, A\&A, 516, A57

Krumholz, M. R., \& McKee, C. F. 2008, Nature, 451, 1082

Leurini, S., Schilke, P., Menten, K. M., et al. 2004, A\&A, 422, 573

Leurini, S., Beuther, H., Schilke, P., et al. 2007a, A\&A, 475, 925

Leurini, S., Schilke, P., Wyrowski, F., \& Menten, K. M. 2007b, A\&A, 466, 215

Looney, L. W., Mundy, L. G., \& Welch, W. J. 2003, ApJ, 592, 255

MacLaren, I., Richardson, K. M., \& Wolfendale, A. W. 1988, ApJ, 333, 821

Mathis, J. S., Rumpl, W., \& Nordsieck, K. H. 1977, ApJ, 217, 425

Müller, H. S. P., Thorwirth, S., Roth, D. A., \& Winnewisser, G. 2001, A\&A, 370, L49

Müller, H. S. P., Schlöder, F., Stutzki, J., \& Winnewisser, G. 2005, J. Mol. Struct., 742,215

Natta, A., Testi, L., Neri, R., Shepherd, D. S., \& Wilner, D. J. 2004, A\&A, 416, 179

Ossenkopf, V., \& Henning, T. 1994, A\&A, 291, 943

Perault, M., Omont, A., Simon, G., et al. 1996, A\&A, 315, L165

Pillai, T., Wyrowski, F., Carey, S. J., \& Menten, K. M. 2006a, A\&A, 450, 569

Pillai, T., Wyrowski, F., Menten, K. M., \& Krügel, E. 2006b, A\&A, 447, 929

Rathborne, J. M., Jackson, J. M., Chambers, E. T., et al. 2005, ApJ, 630, L181

Rathborne, J. M., Jackson, J. M., Zhang, Q., \& Simon, R. 2008, ApJ, 689, 1141

Schuster, K., Boucher, C., Brunswig, W., et al. 2004, A\&A, 423, 1171

Stahler, S. W., \& Palla, F. 2005, The Formation of Stars (Wiley)

Turner, B. E. 1991, ApJS, 76, 617

van der Tak, F. F. S., van Dishoeck, E. F., \& Caselli, P. 2000, A\&A, 361, 327

Wang, Y., Zhang, Q., Rathborne, J. M., Jackson, J., \& Wu, Y. 2006, ApJ, 651, L125

Zhang, Q., Wang, Y., Pillai, T., \& Rathborne, J. 2009, ApJ, 696, 268 